\title{
Online short-term solar power forecasting
}

\author{
Bacher, Peder; Madsen, Henrik; Nielsen, Henrik Aalborg
}

Published in:

Solar Energy

Link to article, DOI:

10.1016/j.solener.2009.05.016

Publication date:

2009

Document Version

Peer reviewed version

Link back to DTU Orbit

Citation (APA):

Bacher, P., Madsen, H., \& Nielsen, H. A. (2009). Online short-term solar power forecasting. Solar Energy, 83(10), 1772-1783. https://doi.org/10.1016/j.solener.2009.05.016

\section{General rights}

Copyright and moral rights for the publications made accessible in the public portal are retained by the authors and/or other copyright owners and it is a condition of accessing publications that users recognise and abide by the legal requirements associated with these rights.

- Users may download and print one copy of any publication from the public portal for the purpose of private study or research.

- You may not further distribute the material or use it for any profit-making activity or commercial gain

- You may freely distribute the URL identifying the publication in the public portal

If you believe that this document breaches copyright please contact us providing details, and we will remove access to the work immediately and investigate your claim. 


\title{
Online Short-term Solar Power Forecasting
}

\author{
Peder Bacher \\ Henrik Madsen \\ Informatics and Mathematical Modelling, Richard Pedersens Plads, Technical University of Denmark \\ Building 321, DK-2800 Lyngby, Denmark \\ Henrik Aalborg Nielsen \\ ENFOR A/S, Lyngsø Allé 3, DK-2970 Hørsholm, Denmark
}

\begin{abstract}
This paper describes a new approach to online forecasting of power production from PV systems. The method is suited to online forecasting in many applications and in this paper it is used to predict hourly values of solar power for horizons of up to 36 hours. The data used is fifteen-minute observations of solar power from 21 PV systems located on rooftops in a small village in Denmark. The suggested method is a two-stage method where first a statistical normalization of the solar power is obtained using a clear sky model. The clear sky model is found using statistical smoothing techniques. Then forecasts of the normalized solar power are calculated using adaptive linear time series models. Both autoregressive (AR) and AR with exogenous input (ARX) models are evaluated, where the latter takes numerical weather predictions (NWPs) as input. The results indicate that for forecasts up to two hours ahead the most important input is the available observations of solar power, while for longer horizons NWPs are the most important input. A root mean square error improvement of around $35 \%$ is achieved by the ARX model compared to a proposed reference model.
\end{abstract}

Key words: Solar power, prediction, forecasting, time series, photovoltaic, numerical weather predictions, clear sky model, quantile regression, recursive least squares

\section{Introduction}

Efforts to increase the capacity of solar power production in Denmark are concentrating on installing grid connected PV sys-

Email addresses: pbac@dtu.dk (Peder Bacher)

$U R L$ : henrikmadsen.org (Henrik Madsen), www.enfor.eu (Henrik Aalborg Nielsen) tems on rooftops. The peak power of the installed PV systems is in the range of 1 to $4 \mathrm{kWp}$, which means that the larger systems will approximately cover the electricity consumption (except heating) of a typical family household in Denmark. The PV systems are connected to the main electricity grid and thus the output from other 


\begin{tabular}{|cll|}
\hline$p$ & Solar power & $\mathrm{W}$ \\
$p_{\mathrm{cs}}$ & Clear sky solar power & $\mathrm{W}$ \\
$\tau$ & Normalized solar power & - \\
$t$ & Time index & - \\
$k$ & Forecast horizon index & - \\
$i, j$ & Miscellaneous indexes & - \\
$p_{t}$ & Observation of average solar power & $\mathrm{W}$ \\
$\hat{p}_{t+k \mid t}$ & $k$-step prediction of solar power & $\mathrm{W}$ \\
$\hat{p}_{t}^{\mathrm{cs}}$ & Estimated clear sky solar power & $\mathrm{W}$ \\
$\hat{g}_{i, k}$ & $i$ 'th update of NWP of global irradiance & $\mathrm{W} / \mathrm{m}^{2}$ \\
$\hat{g}_{k, t}^{00}$ & NWP of global irradiance updated at 00:00 & $\mathrm{W} / \mathrm{m}^{2}$ \\
$\hat{g}_{k, t}^{12}$ & NWP of global irradiance updated at 12:00 & $\mathrm{W} / \mathrm{m}^{2}$ \\
$p_{k, t}^{00}$ & Observation of solar power corresponding to $\hat{g}_{k, t}^{00}$ & $\mathrm{~W}$ \\
$p_{k, t}^{12}$ & Observation of solar power corresponding to $\hat{g}_{k, t}^{12}$ & $\mathrm{~W}$ \\
$\tau_{t}$ & Normalized solar power & - \\
$\hat{\tau}_{t+k \mid t}$ & $k$-step prediction of normalized solar power & - \\
$\hat{\tau}_{t}^{\text {nwp }}$ & NWPs transformed into normalized solar power & - \\
$x_{t}$ & Day of year & - \\
$y_{t}$ & Time of day & - \\
$e_{t+k}$ & $k$-step prediction error & - \\
$q$ & Quantile level & - \\
$h$ & Bandwidth of smoothing kernel & - \\
$\lambda$ & Forgetting factor & - \\
\hline
\end{tabular}

power production units has to be adjusted in order to balance the total power production. The cost of these adjustments increases as the horizon of the adjustments decreases and thus improved forecasting of solar power will result in an optimized total power production, and in future power production systems where energy storage is implemented, power forecasting is an important factor in optimizing utilization of storage facilities (Koeppel and Korpas, 2006).

The total electricity power production in
Denmark is balanced by the energy market Nord Pool, where electricity power is traded on two markets: the main market Elspot and a regulation market Elbas. On Nord Pool the producers release their bids at 12:00 for production each hour the following day, thus the relevant solar power forecasts are updated before 12:00 and consist of hourly values at horizons of 12 to 36 hours. The models in this paper focus on such forecasts, but with the 1-to-11-hour horizons also included. 
Interest in forecasting solar power has increased and several recent studies deal with the problem. Many of these consider forecasts of the global irradiance which is essentially the same problem as forecasting solar power. Two approaches are dominant:

- a two-stage approach in which the solar power (or global irradiance) is normalized with a clear sky model in order to form a more stationary time series and such that the classical linear time series methods for forecasting can be used.

- another approach in which neural networks (NNs) with different types of input are used to predict the solar power (or global irradiance) directly.

In a study Chowdhury and Rahman (1987) make sub-hourly forecasts by normalizing with a clear sky model. The solar power is divided into a clear sky component, which is modelled with a physical parametrization of the atmosphere, and a stochastic cloud cover component which is predicted using ARIMA models. Sfetsos and Coonick (2000) use NNs to make one-step predictions of hourly values of global irradiance and compare these with linear time series models that work by predicting clearness indexes. Heinemann et al. (2006) use satellite images for horizons below 6 hours, and in (Lorenz et al., 2007) numerical weather predictions (NWPs) for longer horizons, as input to NNs to predict global irradiance. This is transformed into solar power by a simulation model of the PV system. Hocaoglu et al. (2008) investigate feed-forward NNs for one-step predictions of hourly values of global irradiance and compare these with seasonal AR models applied on solar power directly. Cao and Lin (2008) use NNs combined with wavelets to predict next day hourly values of global irradiance. Different types of meteorological observations are used as input to the models; among others the daily mean global irradiance and daily mean cloud cover of the day to be forecasted.

This paper describes a new two-stage method where first the clear sky model approach is used to normalize the solar power and then adaptive linear time series models are applied for prediction. Such models are linear functions between values with a constant time difference, where the model coefficients are estimated by minimizing a weighted residual sum of squares. The coefficients are updated regularly, and newer values are weighted higher than old values, hence the models adapt over time to changing conditions.

Normalization of the solar power is obtained by using a clear sky model which gives an estimate of the solar power in clear (non-overcast) sky at any given point in time. The clear sky model is based on statistical smoothing techniques and quantile regression, and the observed solar power is the only input. The adaptive linear prediction is obtained using recursive least squares (RLS) with forgetting. It is found that the adaptivity is necessary, since the characteristics of a PV-system are subject to changes due to snow cover, leaves on trees, dirt on the panel, etc., and this has to be taken into account by an online forecasting system.

The data used in the modelling is described in Section 2. The clear sky model used for normalizing the solar power is defined in Section 3 followed by Section 4 where the adaptive time series models used for prediction are identified. In Section 5 an approach to modelling of the uncertainty in the forecasts is outlined. The evaluation of the models and a discussion of the results 
are found in Section 6 and finally the conclusions of the study are drawn in Section 7

\section{Data}

The data used in this study is observations of solar power from $21 \mathrm{PV}$ systems located in a small village in Jutland, Denmark. The data covers the entire year 2006. Forecasts of global irradiance are provided by the Danish Meteorological Institute using the HIRLAM mesoscale NWP model.

The PV array in each the 21 PV systems is composed of "BP 595" PV modules and the inverters are of the type "BP GCI 1200". The installed peak power of the PV arrays is between 1020 Watt peak and 4080 Watt peak, and the average is 2769 Watt peak. Let $p_{i, t}$ denote the average value of solar power (W) over 15 minutes observed for the $i$ 'th PV system at time $t$. These observations are used to form the time series

$$
\left\{p_{t} ; t=1, \ldots, N\right\}
$$

where

$$
p_{t}=\frac{1}{21} \sum_{i=1}^{21} p_{i, t} .
$$

This time series is used throughout the modelling. The time series covers the period from 01 January 2006 to 31 December 2006. The observations are fifteen-minute values, ie. $N=35040$. Plots of $\left\{p_{t}\right\}$ are shown in Figure 1 for the entire period and for two shorter periods.

The NWPs of global irradiance are given in forecasts of average values for every third hour, and the forecasts are updated at 00:00 and 12:00 each day. The $i$ 'th update of the forecasts is the time series

$$
\left\{\hat{g}_{i, k}, k=1, \ldots, 12\right\}
$$

which then covers the forecast horizons up to 36 hours ahead, and is given in $\left(\mathrm{W} / \mathrm{m}^{2}\right)$.

Time series are resampled to lower sample frequencies by mean values and when the resampled values are used this is noted in the text. In order to synchronize data with different sample frequencies, the time point for a given mean value is assigned to the middle of the period that it covers, e.g. the time point of an hourly value of solar power from 10:00 to 11:00 is assigned to 10:30.

As an example of the NWPs of global irradiance Figure 2 shows values at time of day 10:30 of $\left\{p_{t}\right\}$ resampled to three hour interval values plotted versus the corresponding $\left\{\hat{g}_{i, k}\right\}$ values with a 24 hour horizon. Clearly the plot indicates a significant correlation. Hence it is seen that there is information in the NWPs, which can be utilized to forecast the solar power.

\section{Clear sky model}

A clear sky model is usually a model which estimates the global irradiance in clear (non-overcast) sky at any given time. Chowdhury and Rahman (1987) divide the global irradiance into a clear sky component and a cloud cover component by

$$
G=G_{\mathrm{cS}} \cdot \tau_{c}
$$

where $G$ is the global irradiance $\left(\mathrm{W} / \mathrm{m}^{2}\right)$, and $G_{\mathrm{CS}}$ is the clear sky global irradiance $\left(\mathrm{W} / \mathrm{m}^{2}\right)$. Finally $\tau_{c}$ is the transmissivity of the clouds which they model as a stochastic process using ARIMA models. The clear sky global irradiance is found by

$$
G_{\mathrm{CS}}=I_{0} \cdot \tau_{a}
$$

where $I_{0}$ is the extraterrestrial irradiance $\left(\mathrm{W} / \mathrm{m}^{2}\right) . \quad \tau_{a}$ is the total sky transmissivity in clear sky which is modelled by atmo4 

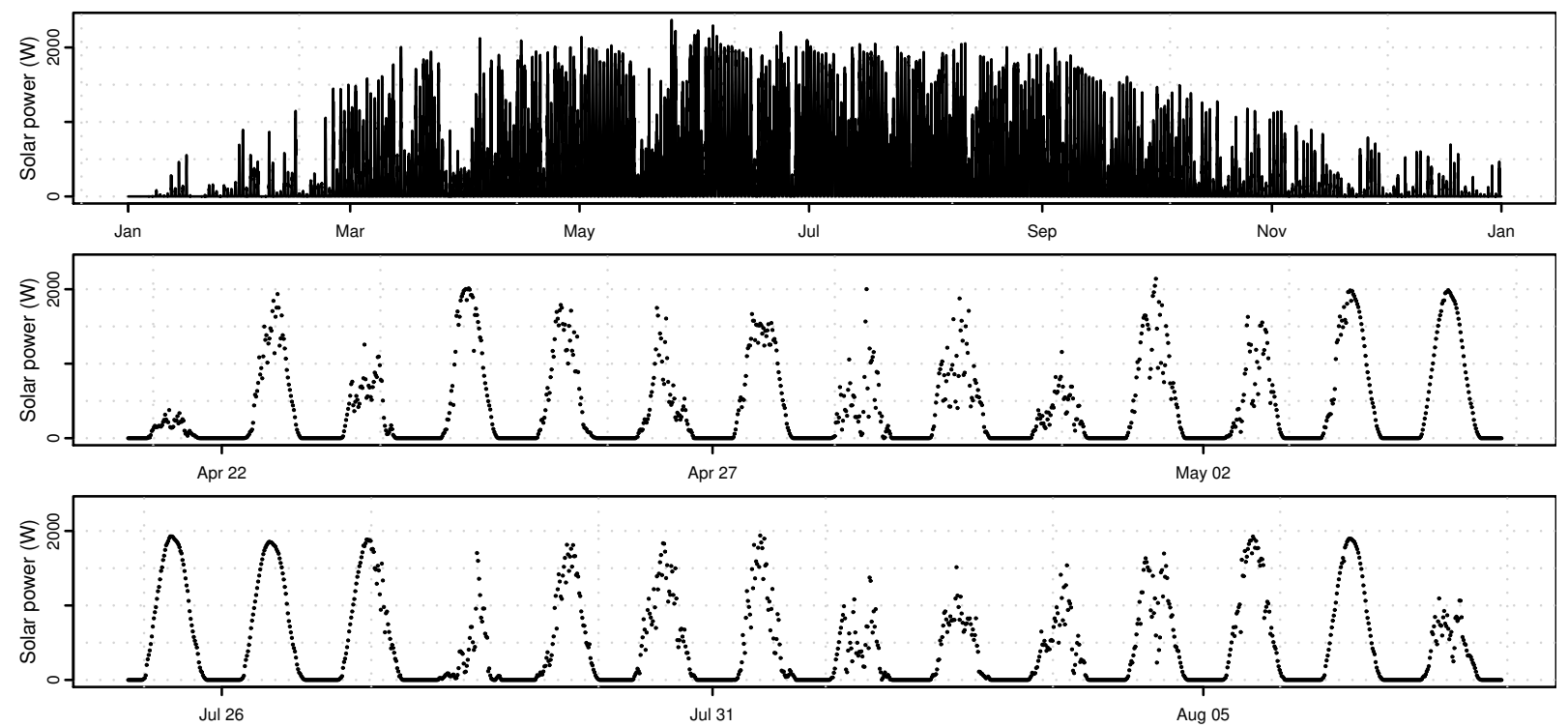

Figure 1: The observations of average solar power used in the study. Upper plot: The solar power over the entire year 2006. Lower plots: The solar power in two selected periods.

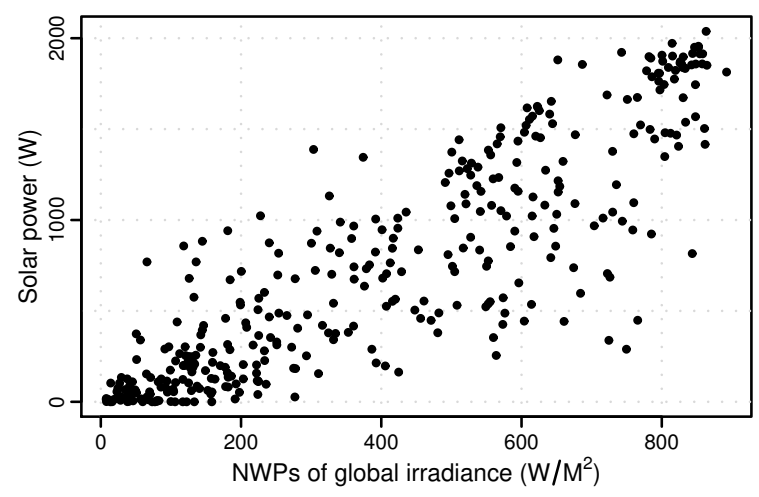

Figure 2: All three hour interval values of solar power at time of day 10:30 versus the corresponding NWPs of global irradiance with 24 hour horizon. Hence the plot shows observations and predictions of values covering identical time intervals.
In this study the same approach is used, but instead of applying the factor on global irradiance it is applied on solar power, i.e.

$$
p=p_{\mathrm{cs}} \cdot \tau
$$

where $p$ is the solar power $(\mathrm{W})$ and $p_{\mathrm{cs}}$ is the clear sky solar power $(\mathrm{W})$. The factors $\tau$ and $\tau_{c}$ are much alike, but since the clear sky model developed in the present study estimates $p_{\text {cs }}$ by statistical smoothing techniques rather than using physics, the method is mainly viewed as a statistical normalization technique and $\tau$ is referred to as normalized solar power.

The motivation behind the proposed normalization of the solar power with a clear sky model is that the normalized solar power (the ratio of solar power to clear sky solar power) is more stationary than the solar power, so that classical time series models assuming stationarity (Madsen, 2007) can be used for predicting the normalized values. The non-stationarity is illustrated by Figure 3 where modified box- 


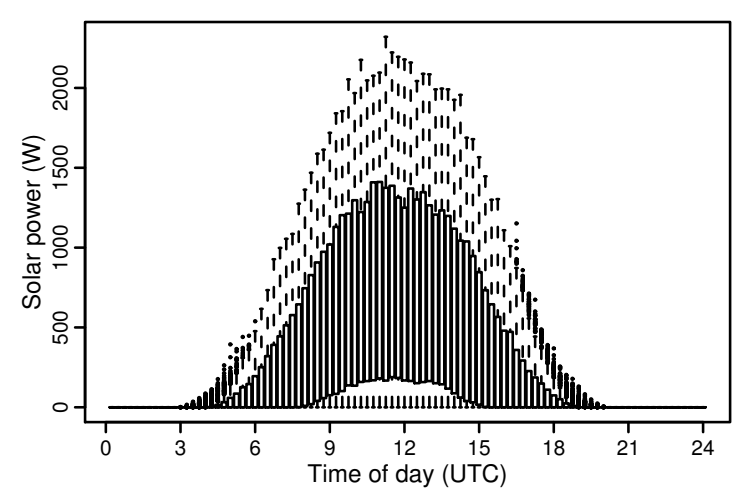

Figure 3: $\quad$ Modified boxplots of the distribution of the solar power as a function of time of day. The boxplots are calculated with all the fifteen-minutes values of solar power, i.e. covering all of 2006. At each time of the day the box represents the center half of the distribution, from the first to the third quantile. The lower and upper limiting values of the distribution are marked with the ends of the vertical dotted lines, and dots beyond these indicate outliers.

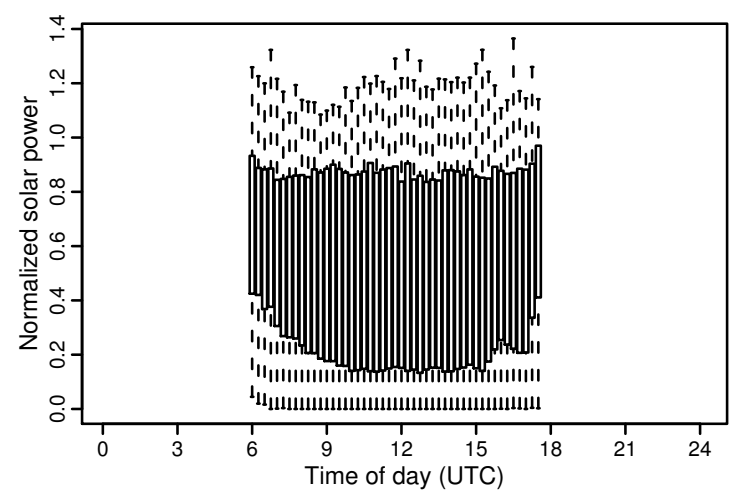

Figure 4: Modified boxplots of the distribution of the normalized solar power as a function of time of day. The boxplots are calculated with all fifteenminutes values available, i.e. covering all of 2006.

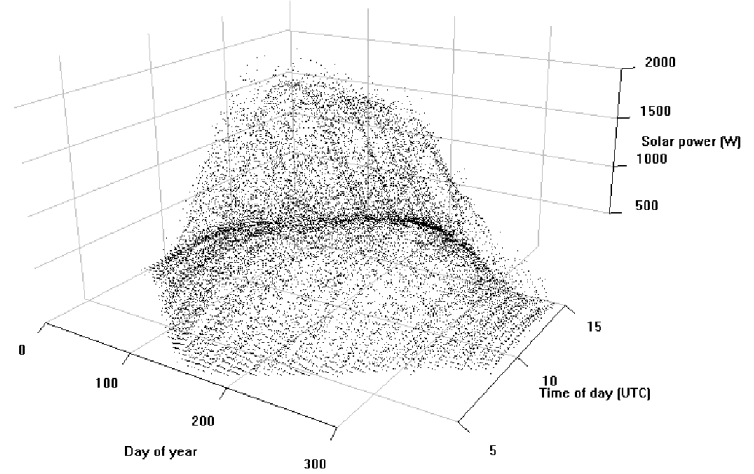

Figure 5: The solar power as a function of the day of year, and the time of day. Note that only positive values of solar power are plotted.

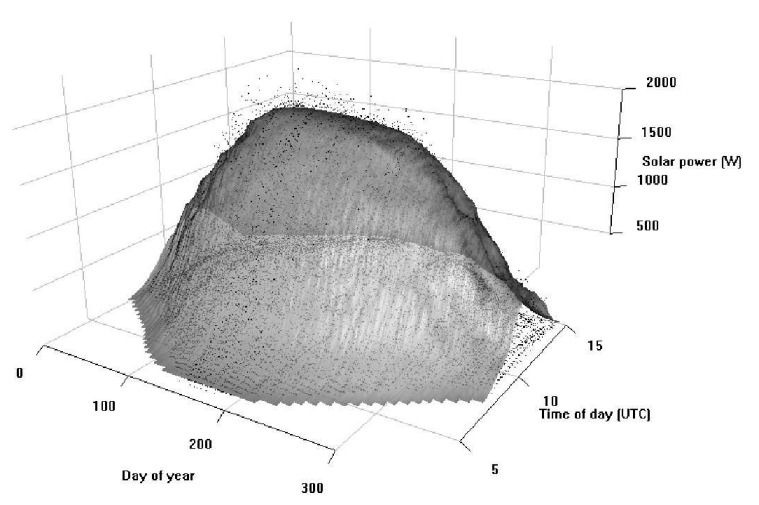

Figure 6: The estimated clear sky solar power shown as a surface. The solar power is shown as points.

plots indicate the distribution of solar power $p_{t}$ as a function of time of day. Clearly a change in the distributions over the day is seen and this non-stationarity must be considered. Figure 4 shows the same type of plot for the normalized solar power and it is seen that the distributions over the day are closer to being identical. Thus the effect of the changes over the day is much lower for the normalized solar power than for the solar power.

The clear sky model is defined as

$$
p_{\mathrm{cs}}=f_{\max }(x, y)
$$



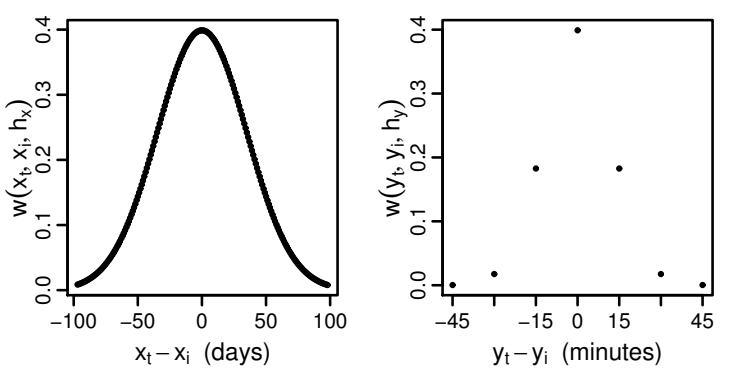

Figure 7: The one dimensional smoothing kernels used. Left plot is the kernel in the day of year (x) dimension. Right plot is the kernel in the time of day (y) dimension. They are multiplied to form the applied two dimensional smoothing kernel.

where $p_{\mathrm{cs}}$ is the clear sky solar power $(\mathrm{W})$, $x$ is the day of year and $y$ is the time of day. The function $f_{\max }(\cdot, \cdot)$ is assumed to be a smooth function and thus $f_{\max }(\cdot, \cdot)$ can be estimated as a local maximum (Koenker, 2005). Figure 5 shows the solar power plotted as a function of $x$ and $y$, and the estimated clear sky solar power $\hat{f}_{\max }(\cdot, \cdot)$ is shown as a surface in Figure 6. Due to outliers the weighted quantile regression method outlined in Appendix $\mathrm{A}$ is used to find the local maximum. The $\hat{f}_{\max }(\cdot, \cdot)$ is then used to form the output of the clear sky model as the time series

$$
\left\{\hat{p}_{t}^{\mathrm{cs}}, t=1, \ldots, N\right\},
$$

where $\hat{p}_{t}^{\text {cs }}$ is the estimated clear sky solar power (W) at time $t$, and $N=35040$. The normalized solar power is now defined as

$$
\tau_{t}=\frac{p_{t}}{\hat{p}_{t}^{\mathrm{cs}}}
$$

and this is used to form time series of normalized solar power

$$
\left\{\tau_{t}, t=1, \ldots, 35040\right\} .
$$

For each $\left(x_{t}, y_{t}\right)$ corresponding to the solar power observation $p_{t}$, weighted quantile regression estimates the $q$ quantile by a Gaussian two dimensional smoothing kernel, defined in Appendix A. The smoothing kernel is used to form the weights applied in the quantile regression. As seen in Figure 7. which shows the smoothing kernel used, the weights in the day of year dimension $w\left(x_{t}, x_{i}, h_{\mathrm{x}}\right)$, are decreasing as the absolute time differences are increasing. Similarly for the weights in the time of day dimension $w\left(y_{t}, y_{i}, h_{\mathrm{y}}\right)$. The applied weights are finally found by multiplying the weigths from the two dimensions. The choice of the quantile level $q$ to be estimated and the bandwidth in each dimension, $h_{\mathrm{x}}$ and $h_{\mathrm{y}}$, is based on a visual inspection of the results. A level of $q=0.85$ was used since this gives $\tau_{t} \approx 1$ for days with clear sky all day, as seen in Figure 8. The plot for days with varying cloud cover in Figure 9 show that estimates where $\tau_{t}>1$ occur. These are ascribed to reflections from clouds and varying level of water vapour in the atmosphere. Future work should elaborate on the inclusion of such effects in the clear sky model.

For small $\hat{p}_{t}^{\text {cs }}$ values the error of $\tau_{t}$ is naturally increasing and at nighttime the error is infinite. Therefore all values of $\hat{p}_{t}^{\text {cs }}$ where

$$
\frac{\hat{p}_{t}^{\mathrm{cs}}}{\max \left(\left\{\hat{p}_{t}^{\mathrm{cs}}\right\}\right)}<0.2
$$

are removed from $\left\{\tau_{t}\right\}$. The function $\max \left(\left\{\hat{p}_{t}^{\text {cs }}\right\}\right)$ gives the maximum value in $\left\{\hat{p}_{t}^{\mathrm{cs}}\right\}$.

The estimates of clear sky solar power are best in the summer period. The bad estimates in winter periods are caused by the sparse number of clear sky observations. It should also be possible to improve the normalization toward dusk and dawn, and thus lower the limit where values in $\left\{\hat{p}_{\mathrm{cs}}\right\}$ are removed, either by refining the modelling 


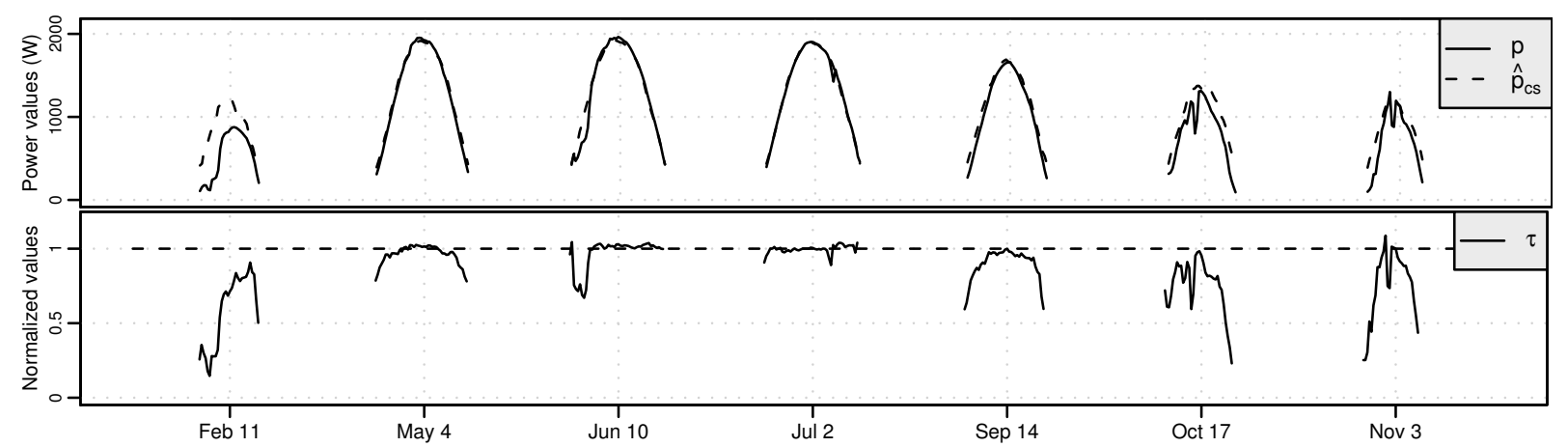

Figure 8: The result of the normalization for selected clear sky days over the year. The time-axis ticks refer to midday points, i.e. at 12:00. The upper plot shows the solar power $p$ and the estimated clear sky solar power $\hat{p}_{\mathrm{cs}}$. The lower plot shows the normalized solar power $\tau$.

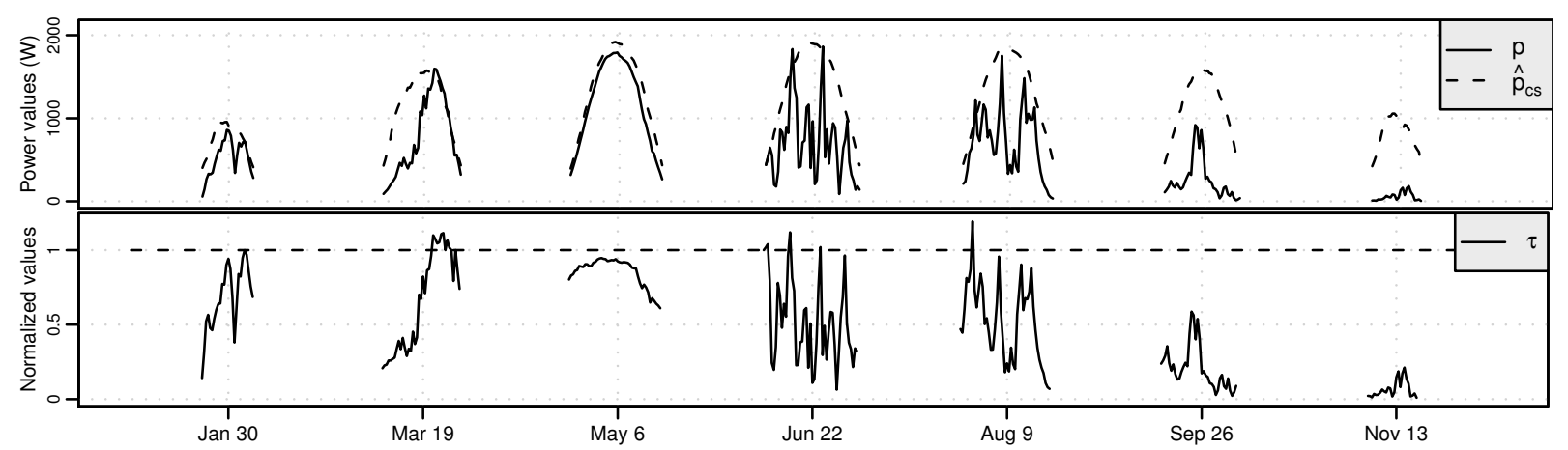

Figure 9: The result of the normalization for days evenly distributed over the year. The time-axis ticks refer to midday points, i.e. at 12:00. The upper plot shows the solar power $p$ and the estimated clear sky solar power $\hat{p}_{\mathrm{cs}}$. The lower plot shows the normalized solar power $\tau$. 
variables such as e.g. air mass.

Finally it is noted that the deterministic changes of solar power are really caused by the geometric relation between the earth and the sun, which can be represented in the current problem by the sun elevation as $x$ and sun azimuth as $y$. The clear sky solar power was also modelled in the space spanned by these two variables, by applying the same statistical methods as for the space spanned by day of year and time of day. The result was not satisfactory, i.e. the estimated clear sky solar power was less accurate, probably because neighboring values in this space are not necessarily close in time and thus changes in the surroundings to the PV system blurred the estimates.

\section{Prediction models}

Adaptive linear time series models $\mathrm{Mad}-$ sen, 2007) are applied to predict future values of the normalized solar power $\tau_{t}$. The inputs are: lagged observations of $\tau_{t}$ and transformed NWPs $\hat{\tau}_{t}^{\text {nwp }}$. Three types of models are identified:

- a model which has only lagged observations of $\tau_{t}$ as input. This is an autoregressive (AR) model and it is referred to as the $A R$ model.

- a model with only $\hat{\tau}_{t}^{\text {nwp }}$ as input. This is referred to as the $L M_{\text {nwp }}$ model.

- a model with both types of input. This is an autoregressive with exogenous input (ARX) model and it is referred to as the $A R X$ model.

The best model of each type is identified by using the autocorrelation function $(\mathrm{ACF})$.

\subsection{Transformation of NWPs into predic- tions of normalized solar power}

In order to use the NWPs of global irradiation $\hat{g}_{i, k}$ as input to the prediction models, these are transformed into $\hat{\tau}_{t}^{\text {nwp }}$ which are meteorological based hourly predictions of $\tau_{t}$. This is done by first transforming $\hat{g}_{i, k}$ into solar power predictions and then transforming these by the clear sky model. The time series $\left\{\hat{g}_{i, k}\right\}$, defined in (3), holds the $i$ 'th NWP forecast of three hour interval values, and was updated at

$$
\text { time }_{i}=t_{0}+(i-1) \cdot 12 \mathrm{~h}
$$

where $t_{0}=2006-01-01$ 00:00. Thus the time series with sample period of one day

$$
\begin{aligned}
\left\{\hat{g}_{k, t}^{00}, t=1, \ldots, 364\right\} & = \\
\left\{\hat{g}_{i, k}, i\right. & =1,3, \ldots, 727\},
\end{aligned}
$$

consist of all the NWPs updated at time of day 00:00 at horizon $k$, i.e. the superscript " 00 " forms part of the name of the variable. Similarly the time series

$$
\begin{aligned}
\left\{\hat{g}_{k, t}^{12}, t=1, \ldots, 364\right\} & = \\
\left\{\hat{g}_{i, k}, i\right. & =2,4, \ldots, 728\},
\end{aligned}
$$

consist of all the NWPs updated at time of day 12:00. The corresponding time series of solar power covering the identical time intervals are respectively

$$
\begin{array}{r}
\left\{p_{k, t}^{00}, t=1, \ldots, 364\right\}= \\
\left\{p_{t}, t=k,(1 \cdot 8+k), \ldots\right. \\
(363 \cdot 8+k)\}
\end{array}
$$

and

$$
\begin{array}{r}
\left\{p_{k, t}^{12}, t=1, \ldots, 364\right\}= \\
\left\{p_{t}, t=k+4,(1 \cdot 8+k+4), \ldots,\right. \\
(363 \cdot 8+k+4)\}
\end{array}
$$


where $\left\{p_{t}\right\}$ has been resampled to three hour interval values. The NWPs are modelled into solar power predictions by the adaptive linear model

$$
\hat{p}_{k, t}^{00}=\beta_{t}+\alpha_{t} \hat{g}_{k, t}^{00}+e_{t},
$$

note that the hat above the variable indicates that these values are predictions (estimates) of the solar power. A similar model is made for the NWP updates at time of day 12:00 giving $\hat{p}_{k, t}^{12}$. The interpretion of the coefficients $\beta_{t}$ and $\alpha_{t}$ is not further elaborated here, but it is noted that they are time dependent in order to account for the effects of changing conditions over time, e.g. the changing geometric relation between the earth and the sun, dirt on the solar panel. This adaptivity is obtained by fitting the model with $k$-step recursive least squares (RLS) with forgetting, which is described in Appendix B. In order to use the RLS algorithm, $p_{k, t}^{00}$ has to be lagged depending on $k$. Each RLS estimation is optimized by choosing the value of the forgetting factor $\lambda$ from $0.9,0.905, \ldots, 1$ that minimizes the root mean square error (RMSE).

The last steps in the transformation of the NWPs is to normalize $\hat{p}_{k, t}^{00}$ and $\hat{p}_{k, t}^{12}$ with the clear sky model, and resample up to hourly values by linear interpolation. Finally the time series

$$
\left\{\hat{\tau}_{t}^{\mathrm{nwp}}, t=1, \ldots, 8760\right\}
$$

of the NWPs of global irradiance transformed into predictions of normalized solar power is formed, and this is used as input to the $A R X$ prediction models as described in the following. More details can be found in (Bacher, 2008).

\subsection{AR model identification}

To investigate the time dependency in $\left\{\tau_{t}\right\}$, i.e. dependency between values with a

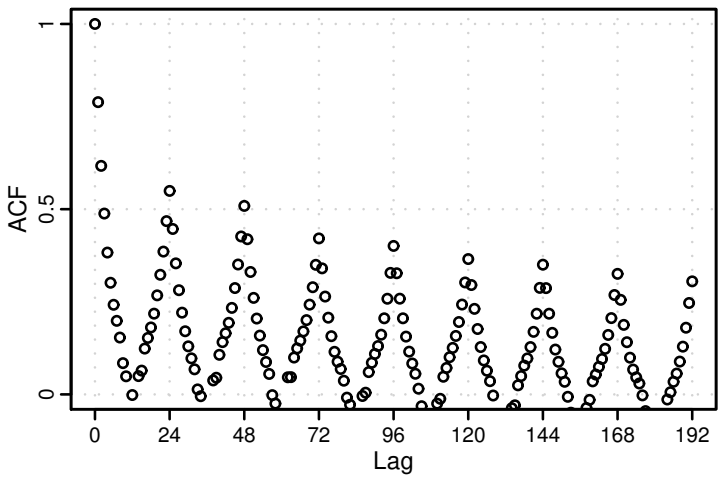

Figure 10: ACF of the time series of normalized solar power $\left\{\tau_{t}\right\}$.

constant time difference, the ACF is calculated and plotted in Figure 10. Clearly an $\mathrm{AR}(1)$ component is indicated by the exponential decaying pattern of the first few lags and a seasonal diurnal AR component by the exponential decaying peaks at lag $=24,48, \ldots$. By considering only first-order terms this leads to the 1-step AR model

$$
\tau_{t+1}=m+a_{1} \tau_{t}+a_{2} \tau_{t-23}+e_{t+1} .
$$

And a reasonable 2-step AR model is

$$
\tau_{t+2}=m+a_{1} \tau_{t}+a_{2} \tau_{t-22}+e_{t+2} .
$$

Note that here the 1-step lag cannot be used, since this is $\tau_{t+1}$ i.e. a future value, and thus the latest observed value is included instead. Formulated as a $k$-step AR model

$$
\begin{aligned}
& \tau_{t+k}=m+a_{1} \tau_{t}+a_{2} \tau_{t-s(k)}+e_{t+k} \\
& s(k)=24+k \bmod 24
\end{aligned}
$$

where the function $s(k)$ ensures that the latest observation of the diurnal component is included. This is needed, since for $k=25$ the diurnal 24 hour AR component cannot be used and instead the 48 hour AR component is used. This model is referred to as the $A R$ model. 

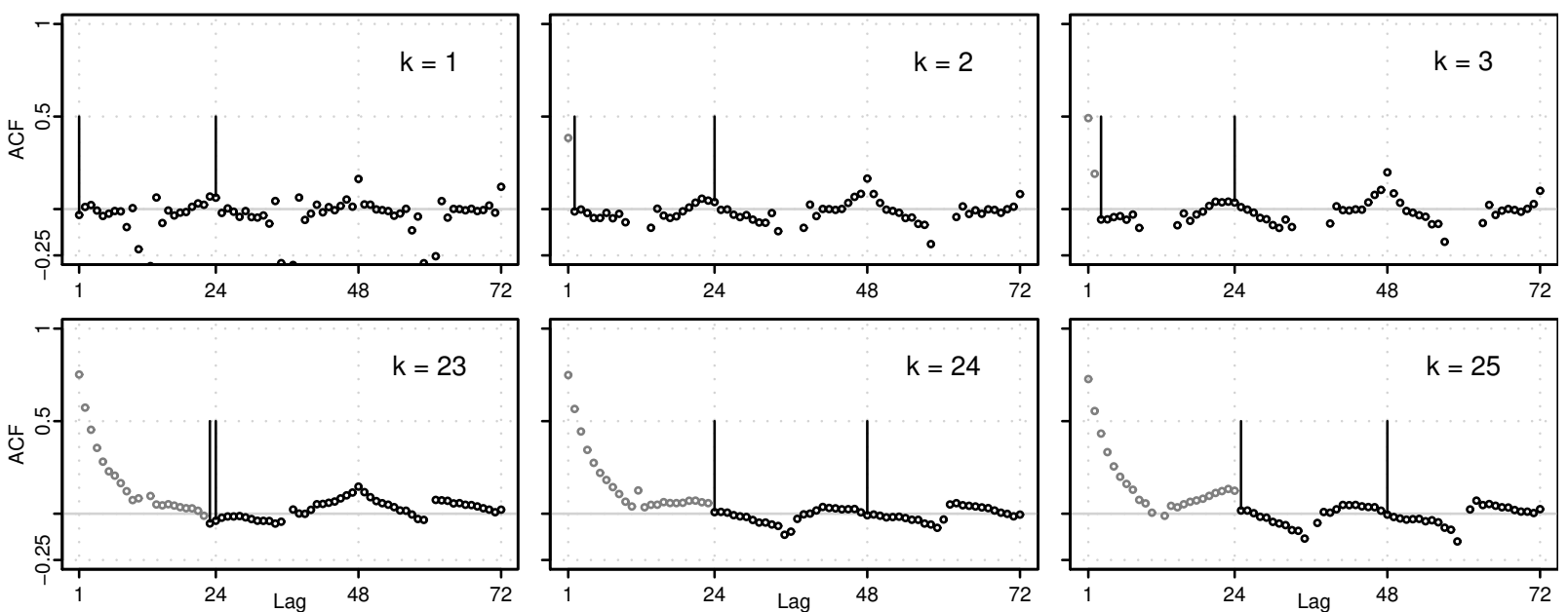

Figure 11: ACF of the time series of errors $\left\{e_{t+k}\right\}$ for selected horizons $k$ of the AR model. The vertical bars indicate the lags included in each of the models, and the grayed points show the lags which cannot be included in the model.

Figure 11 shows the ACF of $\left\{e_{t+k}\right\}$, which is the time series of the errors in the model for horizon $k$, for six selected horizons after fitting the $A R$ model with RLS, which is described in Appendix B. The vertical black lines indicate which lags are included in the model. For $k=1$ the correlation of the $\mathrm{AR}(1)$ component is removed very well and the diurnal AR component has also been decreased considerably. There is high correlation left at lag $=24,48, \ldots$. This can most likely be ascribed to systematic errors caused by non-stationarity effects left in $\left\{\tau_{t}\right\}$, and it indicates that the clear sky model normalization can be further optimized. For $k=2$ and 3 the grayed points show the lags that cannot be included in the model and the high correlation of these lags indicate that information is not exploited. The $A R$ model was extended with higher order $\mathrm{AR}$ and diurnal AR terms without any further improvement in performance, see (Bacher, 2008).
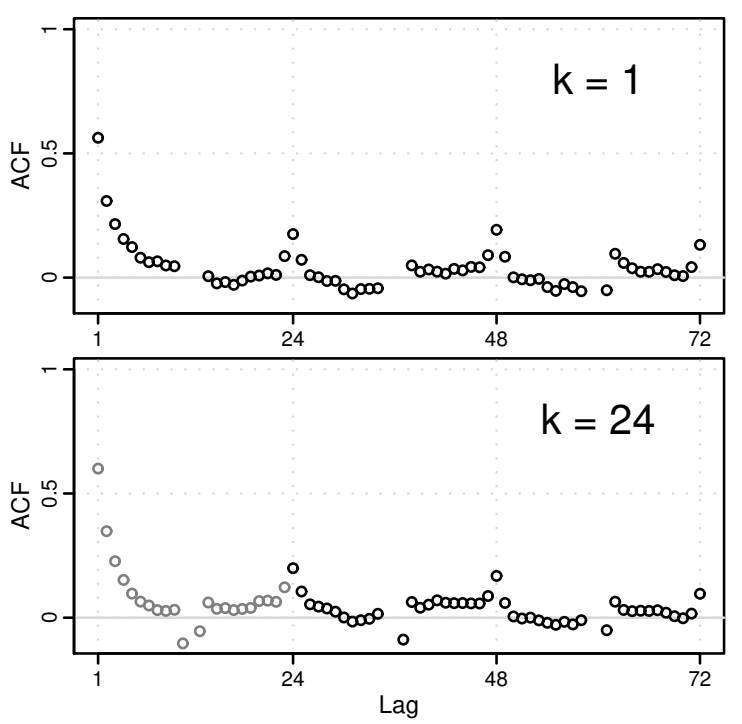

Figure 12: ACF of the time series of errors $\left\{e_{t+k}\right\}$ at horizon $k=1$ and $k=24$ of the $L M_{\text {nwp }}$ model. The grayed points show the lags which cannot be included in the model. 


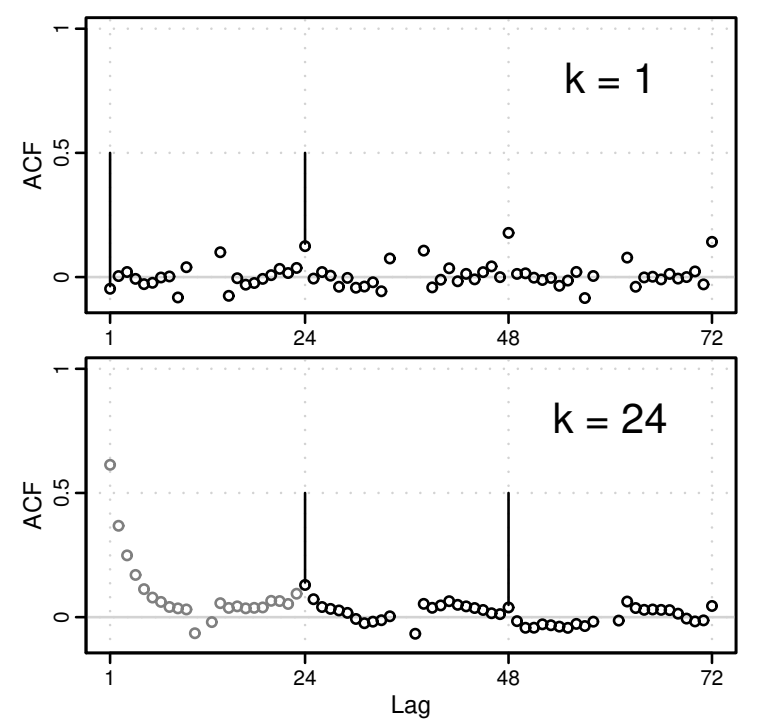

Figure 13: ACF of the time series of errors $\left\{e_{t+k}\right\}$ at horizons $k=1$ and $k=24$ of the ARX model. The vertical bars indicate the lags included in each of the models, and the grayed points show the lags which cannot be included in the model.

\section{3. $L M_{\text {nwp }}$ model identification}

The model using only NWPs as input

$$
\tau_{t+k}=m+b_{1} \hat{\tau}_{t+k \mid t}^{\mathrm{nwp}}+e_{t+k}
$$

is referred to as $L M_{\text {nwp }}$. It is fitted using RLS and the ACF of $\left\{e_{t+k}\right\}$ is shown in Figure 12 for two horizons. For $k=1$ clearly correlation is left from an $\mathrm{AR}(1)$ component, but as seen for both horizons the actual NWP input removes diurnal correlation very well.

\subsection{ARX model identification}

The model using both lagged observations of $\tau_{t}$ and NWPs as input is an ARX model. The $L M_{\text {nwp }}$ revealed an exponential decaying $\mathrm{ACF}$ for short horizons and thus an $\mathrm{AR}(1)$ term is clearly needed, whereas adding the diurnal AR component has only a small effect. The results show that in fact the diurnal AR component can be left out, but it is retained since this clarifies that no improvement is achieved by adding it, this is showed later. The model

$$
\tau_{t+k}=m+a_{1} \tau_{t}+a_{2} \tau_{t-s(k)}+b_{1} \hat{\tau}_{t+k \mid t}^{\mathrm{nwp}}+e_{t+k},
$$

is referred to as the $A R X$ model. The model is fitted using RLS and the ACF of $\left\{e_{t+k}\right\}$ is plotted in Figure 13. It is seen that the AR(1) component removes the correlation for the short horizons very well. The $A R X$ was extended with higher order AR and diurnal AR terms without any further improvements in performance.

\subsection{Adaptive coefficient estimates}

The plots in Figure 14 show the online coefficient estimates for the $A R$ model, where a value of $\lambda=0.995$ is used since this is the value that minimizes the $R M S E_{k}$ best for all horizons in the current setting. Clearly the values of the coefficient estimates change over time and this indicates that the adaptivity is needed to make an optimal model for online forecasting.

\section{Uncertainty modelling}

Extending the solar power forecasts, from predicting a single value (a point forecast) to predicting a distribution increases their usefulness. This can be achieved by modelling the uncertainties of the solar power forecasts and a simple approach is outlined here. The classical way of assuming normal distribution of the errors will in this case not be appropriate since the distribution of the errors has finite limits. Instead, quantile regression is used, inspired by Møller et al. (2008) where it is applied to wind power forecasts. Plots of $\left\{\tau_{t}\right\}$ versus $\left\{\hat{\tau}_{t}\right\}$ for a given horizon reveal that the uncertainties depend on the level of $\hat{\tau}$. Figure 

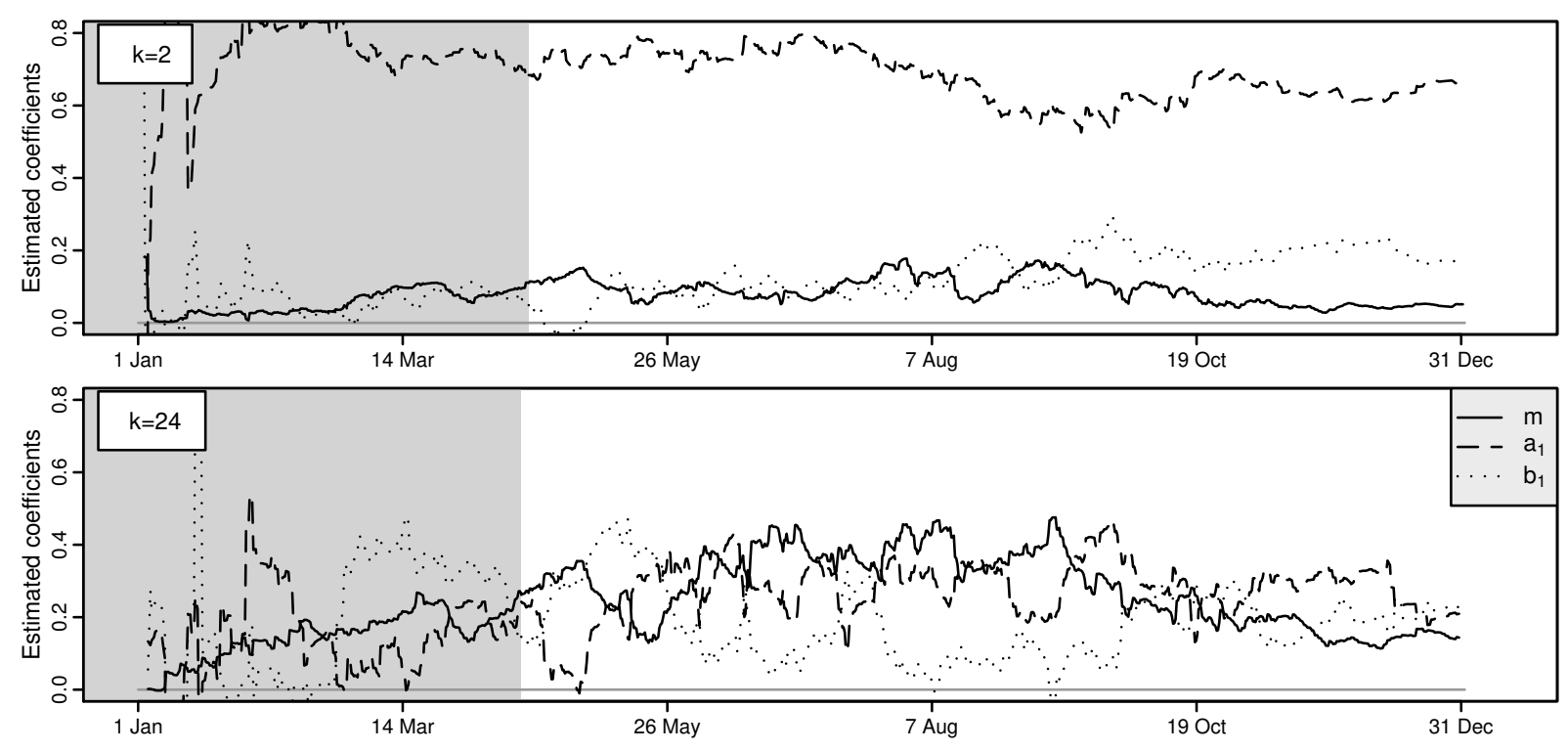

Figure 14: The online estimates of the coefficients in the AR model as a function of time. Two selected horizons are shown. The grayed period in the beginning marks the burn-in period.
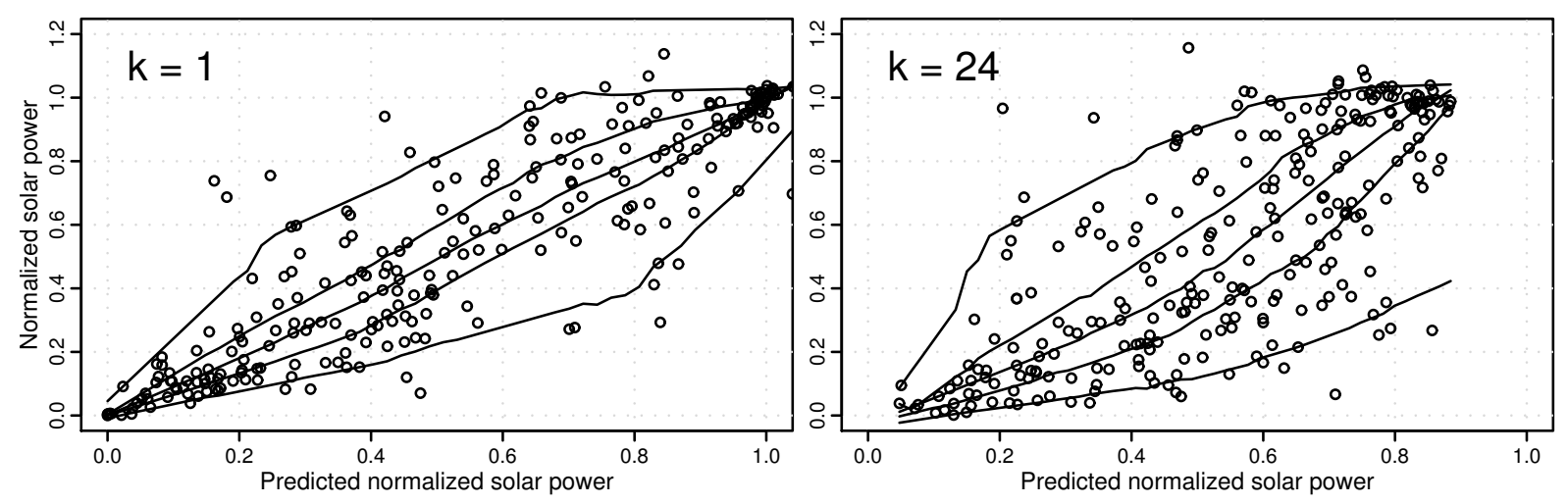

Figure 15: $\quad$ Normalized solar power versus the predicted normalized solar power at horizons $k=1$ and $k=24$. The predictions are made with the ARX model. The lines are estimates of the 0.05, 0.25, 0.50,0.75 and 0.95 quantiles of $f_{\tau}(\hat{\tau})$. 
15 shows such plots for horizons $k=1$ and $k=24$. The lines in the plot are estimates of the $0.05,0.25,0.50,0.75$ and 0.95 quantiles of the probability distribution function of $\tau$ as a function of $\hat{\tau}$. The weighted quantile regression with a one dimensional kernel smoother, described in Appendix A, is used.

Figure 15 illustrates that the uncertainties are lower for $\hat{\tau}$ close to 0 and 1 , than for the mid-range values around 0.5. Thus forecasts of values toward overcast or clear sky have less uncertainty than forecasts of a partly overcast sky, which agrees with results by Lorenz et al. (2007). Further work should extend the uncertainty model to include NWPs as input.

\section{Evaluation}

The methods used for evaluating the prediction models are inspired by Madsen et al. (2005) where a framework for evaluation of wind power forecasting is suggested. The RLS fitting of the prediction models does not use any degrees of freedom and the dataset is therefore not divided into a training set and a test set. It is, however, noted that the clear sky model and the optimization of $\lambda$ does use the entire dataset, and thus the results can be a little optimistic. The values in the burn-in period are not used in calculating the error measures. In Figure 14 the burn-in periods for the $A R$ model are shown.

\subsection{Error measures}

The $k$-step prediction error is

$$
e_{t+k}=p_{t+k}-\hat{p}_{t+k \mid t}
$$

The Root Mean Square Error for the $k$ 'th horizon is

$$
R M S E_{k}=\left(\frac{1}{N} \sum_{t=1}^{N} e_{t+k}^{2}\right)^{\frac{1}{2}}
$$

The $R M S E_{k}$ is used as the main evaluation criterion (EC) for the performance of the models. The Normalized Root Mean Square Error is found by

$$
N R M S E_{k}=\frac{R M S E_{k}}{p_{\text {norm }}}
$$

where either

$$
p_{\text {norm }}=\bar{p}=\frac{1}{N} \sum_{t=1}^{N} p_{t} .
$$

or $p_{\text {norm }}$ is the average peak power of the 21 PV systems.

The mean value of the $R M S E_{k}$ for a range of horizons

$$
\overline{R M S E}_{k_{\mathrm{s}}, k_{\mathrm{e}}}=\frac{1}{k_{\mathrm{e}}-k_{\mathrm{s}}+1} \sum_{k=k_{\mathrm{s}}}^{k_{\mathrm{e}}} R M S E_{k}
$$

is used as a summary error measure. When comparing the performance of two models the improvement

$$
I_{\mathrm{EC}}=100 \cdot \frac{E C_{\mathrm{ref}}-E C}{E C_{\mathrm{ref}}}(\%)
$$

is used, where $E C$ is the considered evaluation criterion.

\subsection{Reference model}

To compare the performance of prediction models, and especially when making comparisons between different studies, a common reference model is essential. A reference model for solar power is here proposed as the best performing naive predictor for the given horizon. Three naive predictors of solar power are found to be relevant. Persistence

$$
p_{t+k}=p_{t}+e_{t+k},
$$




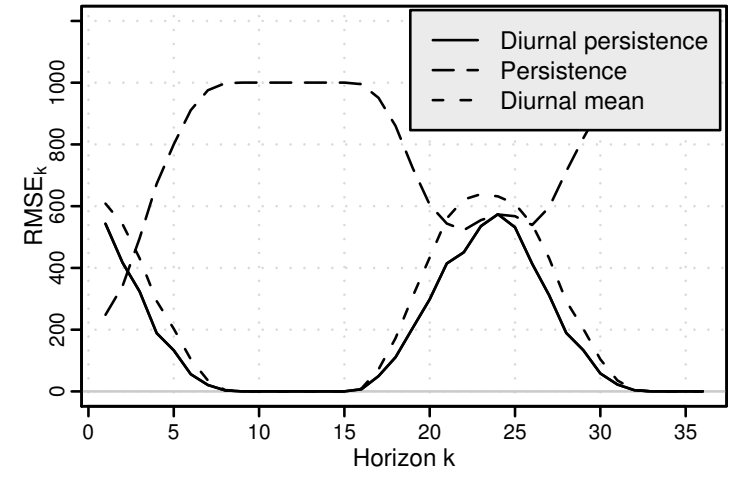

Figure 16: $R M S E_{k}$ for the three naive predictors used in the Reference model.

diurnal persistence

$p_{t+k}=p_{t-s(k)}+e_{t+k}$

$s(k)=f_{\mathrm{spd}}+k \bmod f_{\mathrm{spd}}$

where $s(k)$ ensures that the latest diurnal observation is used and $f_{\text {spd }}$ is the sample frequency in number of samples per day, and diurnal mean

$$
\begin{aligned}
p_{t+k} & =\frac{1}{n} \sum_{i=1}^{n} p_{t-s(k, i)}+e_{t+k} \\
s(k, i) & =i \cdot f_{\mathrm{spd}}+k \bmod f_{\mathrm{spd}}
\end{aligned}
$$

which is the mean of solar power of the last $n$ observations at the time of day of $p_{t+k}$. The value of $n$ is chosen such that all past samples are included.

Figure 16 shows the $R M S E_{k}$ for each of the three naive predictors. It is seen that for $k \leq 2$ the persistence predictor is the best while the best for $k>2$ is the diurnal persistence predictor. This model is referred to as the Reference model.

\subsection{Results}

Examples of solar power forecasts made with the $A R X$ model are shown in Figure 17 for short horizons and in Figure 18 for next day horizons. It is found that the forecasted solar power generally follows the main level of the solar power, but the fluctuations caused by sudden changes in cloud cover are not fully described by the model.

The $N R M S E_{k}$ is plotted for each model in Figure 19. Clearly the performance is increasing from the Reference model to the $A R$ model and further to the $A R X$ model. The differences from using either the solar power or the NWPs, or both, as input become apparent from these results.

At $k=1$ the $A R$ model that only uses solar power as input is better than the $L M_{\text {nwp }}$ which only uses NWPs as input, but at $k=$ $2, \ldots, 6$ the $L M_{\text {nwp }}$ is better, though only slightly. This indicates that for making forecasts of horizons shorter than 2 hours, solar power is the most important input, whereas for 2 to 6 hours horizons, forecasting systems using either solar power or NWPs can perform almost equally. The $A R X$ model using both types of input does have an increased performance at all $k=1, \ldots, 6$ and thus combining the two types of input is found to be the superior approach.

For $k=19, \ldots, 29$, which are the next day horizons, very clearly the $L M_{\text {nwp }}$ model and the $A R X$ model perform better than the $A R$ model. Since the $L M_{\text {nwp }}$ model and the $A R X$ model perform almost equally, it is seen that no improvement is achieved from adding the solar power as input, and thus using only the NWPs as input is found to be adequate for next day horizons.

A summary of the improvement in performance is calculated using (29) and (30). The improvements compared to the Reference model are calculated for the four models by $I_{\overline{R M S E}_{1,6}}$ for short horizons and $I_{\overline{R M S E}_{19,29}}$ for next day horizons. The results are shown in Table 1. These results naturally show the same as stated above, though the difference at $k=1$ from $A R$ to 


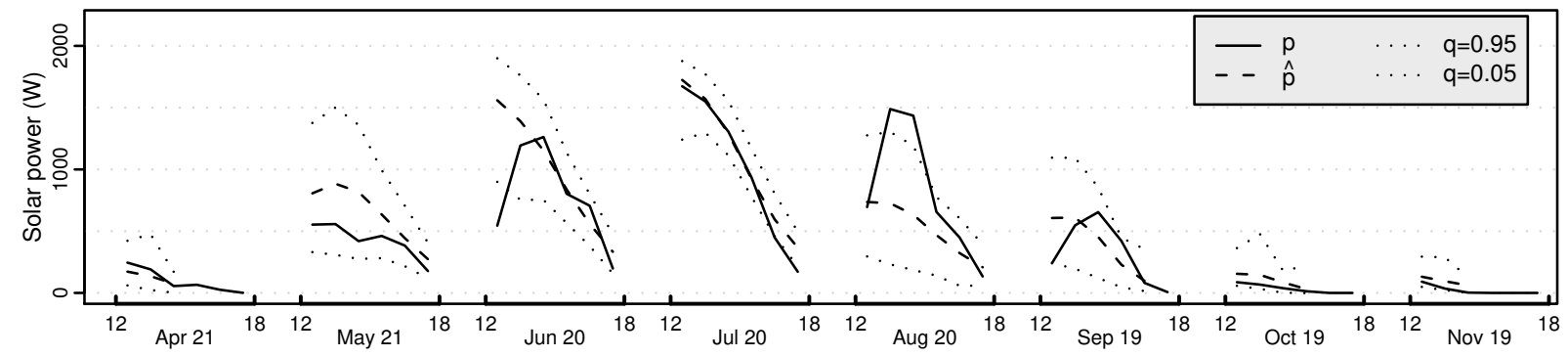

Figure 17: Forecasts of solar power at short horizons $k=1, \ldots, 6$ made with the ARX model.

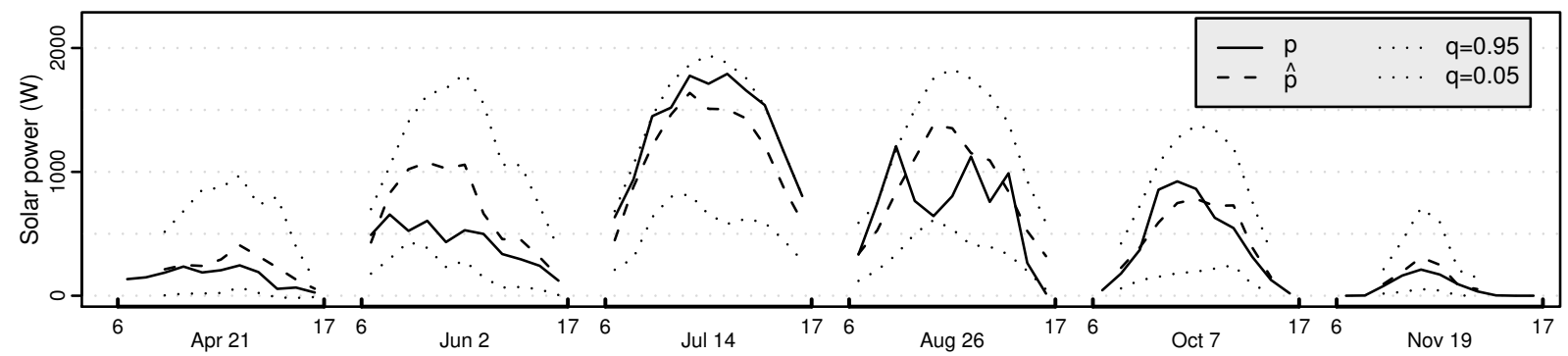

Figure 18: Forecasts of solar power at next day horizons $k=19, \ldots, 29$ made with the ARX model.

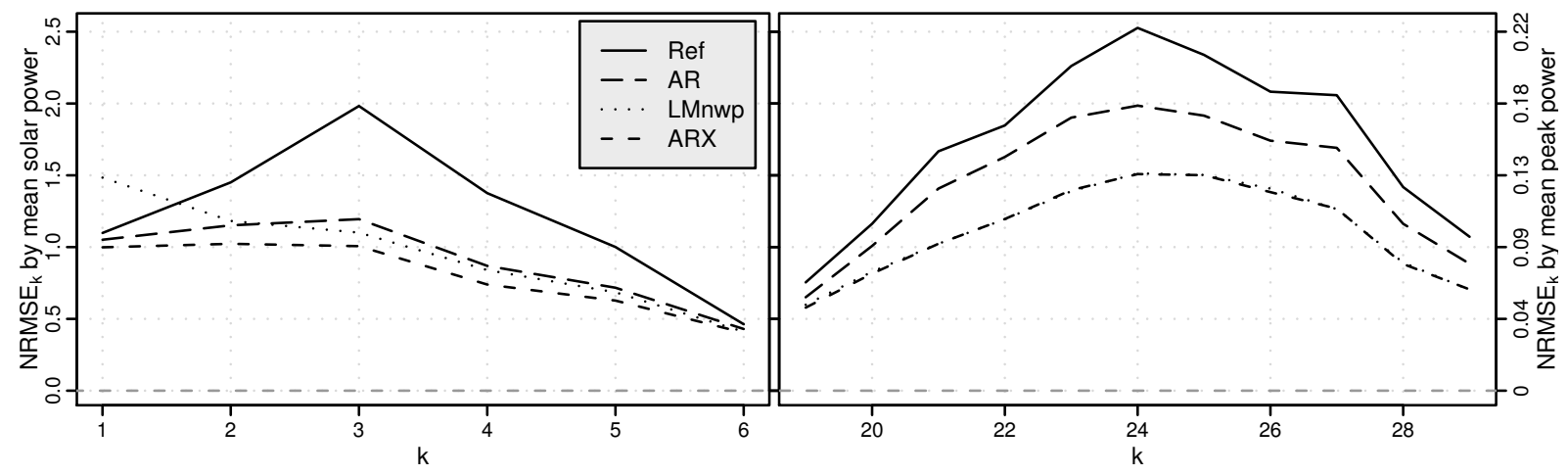

Figure 19: The NRMSE $E_{k}$ for each of the three models and the Reference model. The left plot show the short horizons and the right the next day horizons. The left scale show $R M S E_{k}$ normalized by $\bar{p}=248 \mathrm{~W} / \mathrm{h}$ and the right scale show $R M S E_{k}$ normalized by $2769 \mathrm{~W}$ which is the mean peak power of the 21 PV systems. 


\begin{tabular}{ccc}
\hline Models & $I_{\overline{R M S E}_{1,6}}$ & $I_{\overline{R M S E}_{19,29}}$ \\
\hline$A R$ over Reference & $27 \%$ & $17 \%$ \\
$L M_{\text {nwp }}$ over Reference & $25 \%$ & $36 \%$ \\
$A R X$ over Reference & $35 \%$ & $36 \%$ \\
\hline$L M_{\text {nwp }}$ over $A R$ & $-2 \%$ & $23 \%$ \\
$A R X$ over $A R$ & $12 \%$ & $23 \%$ \\
\hline$A R X$ over $L M_{\text {nwp }}$ & $13 \%$ & $1 \%$ \\
\hline
\end{tabular}

Table 1: Summary error measures of improvements compared to the Reference model for short horizons $k=1, \ldots, 6$ and next day horizons $k=19, \ldots, 29$.

$L M_{\text {nwp }}$ cannot be seen. These results show that a RMSE improvement of around $35 \%$ over the Reference model can be achieved by using the $A R X$ model.

\section{Conclusions}

Inspired by previous studies, the present method for solar power forecasting has been developed from scratch. A new approach to clear sky modelling with statistical smoothing techniques has been proposed, and an adaptive prediction model based on RLS makes a solid framework allowing for further refinements and model extensions e.g. by including NWPs of temperature as input. The adaptivity of the method makes it suited to online forecasting and ensures comprehension of changing conditions of the PV system and its surroundings. Furthermore the RLS algorithm is not computer intensive, which makes updating of forecasts fast. The clear sky model used to normalize the solar power delivers a useful result, but can be improved, especially for the estimates toward dawn and dusk, by using polynomial-based kernel regression. A procedure based on quantile regression is suggested for calculating the varying intervals of the uncertainty of the solar power predictions and the results agree with other stud- ies. The best performing prediction model is an ARX model where both solar power observations and NWPs are used as input. The results indicate that for horizons below 2 hours solar power is the most important input, but for next day horizons no considerable improvement is achieved from using available values of solar power, so it is adequate just to use NWPs as input. Thus, depending on the application of the forecasting system using only either of the inputs can be considered, and a lower limit of the latency, at which solar power observations are needed for the forecasting system, can be different. Finally it is noted that a comparison to other online solar power forecasting methods, e.g. (Lorenz et al., 2007) and (Hocaoglu et al., 2008), has not been carried out, but that such a study would be informative in order to describe strengths and accuracy of the different proposed methods.

\section{A. Weighted quantile regression}

The solar power time series $\left\{p_{t}, t=\right.$ $1, \ldots, N\}$ is the realization of a stochastic process $\left\{P_{t}, t=1, \ldots, N\right\}$. The estimated clear sky solar power at time $t$ is $\hat{p}_{t}^{\mathrm{cs}}$ and it is found as the $q$ quantile of $f_{P_{t}}$, the probability distribution function of $P_{t}$. The problem is reduced to estimating $\hat{p}_{t}^{\text {cs }}$ as a local constant for each $\left(x_{t}, y_{t}\right)$, where $x$ is the day of year and $y$ the time of day. This is done by weighted quantile regression in which the loss function is

$$
\rho\left(q, \epsilon_{i}\right)=\left\{\begin{array}{lll}
q \epsilon_{i} & , & \epsilon_{i} \geq 0 \\
(1-q) \epsilon_{i} & , & \epsilon_{i}<0
\end{array}\right.
$$

where $\epsilon_{i}=p_{i}-\hat{p}_{t}^{\mathrm{cs}}$. The fitting of $\hat{p}_{t}^{\mathrm{cs}}$ is then done by 
where

$$
Y_{t}=\mathbf{X}_{t}^{T} \theta+e_{t} .
$$

$k\left(x_{t}, y_{t}, x_{i}, y_{i}\right)=\frac{w\left(x_{t}, x_{i}, h_{\mathrm{x}}\right) \cdot w\left(y_{t}, y_{i}, h_{\mathrm{y}}\right)}{\sum_{i=1}^{N} w\left(x_{t}, x_{i}, h_{\mathrm{x}}\right) \cdot w\left(y_{t}, y_{i}, h_{\mathrm{y}}\right)}$ The estimates of the parameters at $t$ are

is the two dimensional multiplicative kernel function which weights the observations locally to $\left(x_{t}, y_{t}\right)$, (Hastie and Tibshirani, 1993). Details of the minimization are found in (Koenker, 2005). In each dimension a Gaussian kernel is used

$$
w\left(x_{t}, x_{i}, h_{x}\right)=f_{\text {std }}\left(\frac{\left|x_{t}-x_{i}\right|}{h_{x}}\right)
$$

where $f_{\text {std }}$ is the standard normal probability density function. A similar kernel function is used in the $y$ dimension, and the final two dimensional kernel is found by multiplying the two kernels as shown in (37).

\section{B. Recursive least squares}

Fitting of the prediction models is done using $k$-step recursive least squares (RLS) with forgetting, which is described in the following using the $A R X$ model

$$
\tau_{t+k}=m+a_{1} \tau_{t}+a_{2} \tau_{t-s(k)}+b_{1} \hat{\tau}_{t+k \mid t}^{\text {nwp }}+e_{t+k}
$$

as an example. The regressor at time $t$ is

$$
\mathbf{X}_{t}^{T}=\left(1, \tau_{t}, \tau_{t-s(k)}, \hat{\tau}_{t+k \mid t}^{\mathrm{nwp}}\right),
$$

the parameter vector is

$$
\theta^{T}=\left(m, a_{1}, a_{2}, b_{1}\right),
$$

and the dependent variable

$$
Y_{t}=\tau_{t} .
$$

$$
\hat{\theta}_{t}=\arg \min _{\theta} S_{t}(\theta),
$$

where the loss function is

$$
S_{t}(\theta)=\sum_{s=1}^{t} \lambda^{t-s}\left(Y_{s}-\mathbf{X}_{s}^{T} \theta\right)^{2} .
$$

This provides weighted least squares with exponential forgetting. The solution at time $t$ leads to

$$
\hat{\theta}_{t}=\mathbf{R}_{t}^{-1} \mathbf{h}_{t}
$$

see (Madsen, 2007), where

$$
\mathbf{R}_{t}=\sum_{s=1}^{t} \lambda^{t-s} \mathbf{X}_{s} \mathbf{X}_{s}^{T}, \quad \mathbf{h}_{t}=\sum_{s=1}^{t} \lambda^{t-s} \mathbf{X}_{s} Y_{s} .
$$

The $k$-step RLS-algorithm with exponential forgetting is then

$$
\begin{aligned}
\mathbf{R}_{t} & =\lambda \mathbf{R}_{t-1}+\mathbf{X}_{t-k} \mathbf{X}_{t-k}^{T} \\
\hat{\theta}_{t} & =\hat{\theta}_{t-1}+\mathbf{R}_{t}^{-1} \mathbf{X}_{t-k}\left(Y_{t}-\mathbf{X}_{t-k}^{T} \hat{\theta}_{t-1}\right)
\end{aligned}
$$

and the $k$-step prediction at $t$ is

$$
\hat{Y}_{t+k}=\mathbf{X}_{t}^{T} \hat{\theta}_{t}
$$

\section{References}

Bacher, P., 2008. Short-term solar power forecasting. Master's thesis, Technical University of Denmark, IMM-M.Sc.-2008-13.

Cao, J., Lin, X., 2008. Study of hourly and daily solar irradiation forecast using diagonal recurrent wavelet neural networks. Energy Conversion and Management 49 (6), 1396-1406. 
Chowdhury, B., Rahman, S., 1987. Forecasting subhourly solar irradiance for prediction of photovoltaic output. In: IEEE Photovoltaic Specialists Conference, 19th, New Orleans, LA, May 4-8, 1987, Proceedings (A88-34226 13-44). New York, Institute of Electrical and Electronics Engineers, Inc., 1987, p. 171-176. pp. 171-176.

Hastie, T., Tibshirani, R., 1993. Varying-coefficient models. Journal of the Royal Statistical Society. Series B (Methodological) 55 (4), 757-796.

Heinemann, D., Lorenz, E., Girodo, M., 2006. Forecasting of solar radiation. In: Dunlop, E., Wald, L., Suri, M. (Eds.), Solar Resource Management for Electricity Generation from Local Level to Global Scale. Nova Science Publishers, New York, pp. 83-94.

Hocaoglu, F. O., Gerek, Ö. N., Kurban, M., 2008. Hourly solar radiation forecasting using optimal coefficient 2-d linear filters and feed-forward neural networks. Solar Energy 82 (8), 714-726.

Koenker, R., 2005. Quantile Regression. Cambridge University Press.

Koeppel, G., Korpas, M., 2006. Using storage devices for compensating uncertainties caused by non-dispatchable generators. 2006 International Conference on Probabilistic Methods Applied to Power Systems, 1-8.

Lorenz, E., Heinemann, D., Wickramarathne, H., Beyer, H., Bofinger, S., 2007. Forecast of ensemble power production by grid-connected pv systems. In: Proc. 20th European PV Conference, September 3-7, 2007, Milano.

Madsen, H., 2007. Time Series Analysis. Chapman \& Hall.

Madsen, H., Pinson, P., Kariniotakis, G., Nielsen, H. A., Nielsen, T. S., 2005. Standardizing the performance evaluation of shortterm wind power prediction models. Wind Engineering 29 (6), 475.

Møller, J. J. K., Nielsen, H. A., Madsen, H., 2008. Time-adaptive quantile regression. Computational Statistics and Data Analysis 52 (3), 1292-1303.

Sfetsos, A., Coonick, A., 2000. Univariate and multivariate forecasting of hourly solar radiation with artificial intelligence techniques. Solar Energy 68 (2), 169-178. 